\title{
Nonlethal Raine Syndrome in a Newborn Boy Caused by a Novel FAM20C Variant
}

\author{
Nazan Eras ${ }^{\mathrm{a}}$ Yalcin Celik ${ }^{\mathrm{b}}$

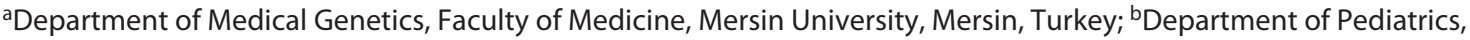 \\ Division of Neonatology, Faculty of Medicine, Mersin University, Mersin, Turkey
}

\section{Established Facts}

- Raine syndrome is a rare genetic condition caused by a homozygous mutation, compound heterozygous mutation, or microdeletion in the FAM20C gene.

- Clinical features include generalized osteosclerosis, intracranial calcification, choanal atresia/stenosis, dysmorphic face, and sensorineural hearing loss.

\section{Novel Insights}

- The c.1255T >C variant p.(W419R) is the first missense variant reported in nonlethal Raine syndrome.

\section{Keywords}

Choanal stenosis · FAM20C · Osteosclerosis · Raine syndrome · Variant

\begin{abstract}
Raine syndrome (RS) is a rare genetic disorder characterized by osteosclerotic bone dysplasia caused by a homozygous mutation, compound heterozygous mutation, or microdeletion in the FAM20C gene. In the present study, the MiSeq next-generation sequencing platform was used to perform the FAM20C gene sequence analysis. A novel homozygous variant c.1255T>C (p.W419R) in the FAM20C gene was diagnosed, and a nonlethal RS phenotype was confirmed, thus contributing to the expansion of the nonlethal RS phenotype. Since there is limited information about rare diseases,
\end{abstract}

we believe that these studies will contribute to the literature and to the understanding of how these disorders develop and progress.

(c) 2021 S. Karger AG, Base

\section{Introduction}

The first lethal Raine syndrome (RS; OMIM \#259775) was described by Raine et al. [1989] in a newborn with a small nose, underdevelopment of the upper jaw, cleft palate, low-set ears, exophthalmos, microcephaly, micrognathism, and neonatal osteosclerotic bone dysplasia. It is caused by a homozygous mutation, compound heterozygous mutation, or microdeletion in the FAM20C gene $\left({ }^{*} 611061\right)$ at chromosome $7 \mathrm{p} 22.3$, with an estimated 


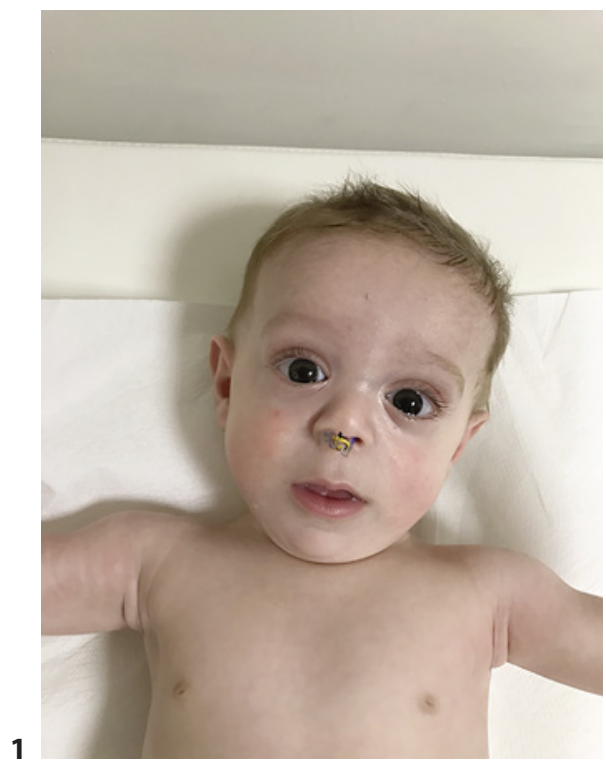

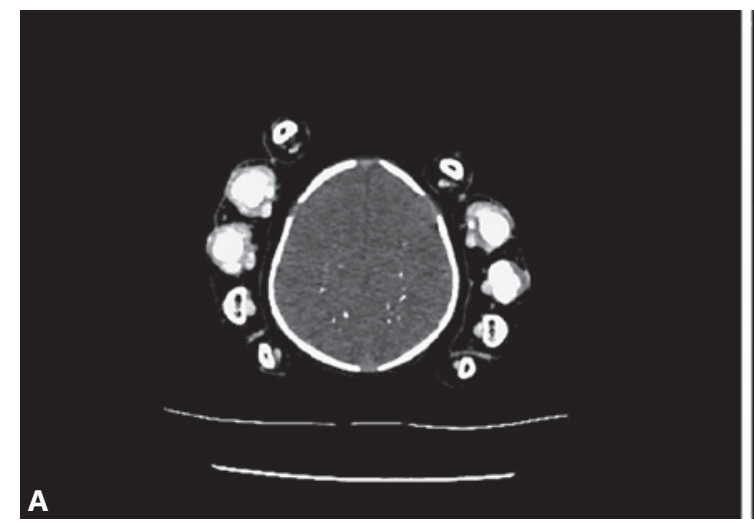

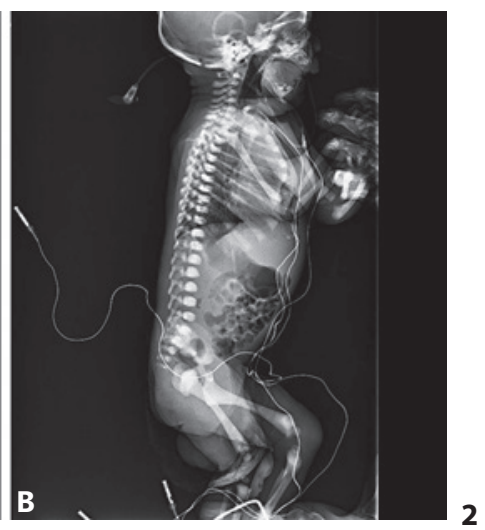

Fig. 1. Clinical photograph of the patient with nonlethal RS showing pectus excavatum and distinct facial features including underdeveloped midface, flattened forehead, epicanthal fold, anteverted nares, small nose, depressed nasal bridge, and small mouth.

Fig. 2. Noncontrast brain CT and direct radiography at the age of 1 month. A The brain CT shows that there are numerous microcalcifications in both hemispheres. B Diffuse osteosclerosis shown in the skull base, vertebra, and long bones on direct radiography. prevalence of $<1$ in 1,000,000 [Simpson et al., 2009; Faundes et al., 2014]. The disorder was defined as neonatal osteosclerotic bone dysplasia, generally resulting in death in the first few weeks of life, until 2009. After being described in 2 patients with sclerosing bone dysplasia at the age of 8 and 11 years, the nonlethal type of lethal RS had begun to be reported [Simpson et al., 2009]. Here, we present a case of a newborn boy with nonlethal RS, who had a novel homozygous variant c.1255T >C (p.W419R) and whose parents had a heterozygous variant.

\section{Case Report}

A 1-day-old male neonate was referred to our hospital from an external center with the suspicion of choanal atresia due to respiratory distress and failure to advance the nasogastric tube through both nostrils. He weighed 3,260 $\mathrm{g}$ (25th-50th percentile); length and head circumference were $47 \mathrm{~cm}$ (10th-25th percentile) and $33 \mathrm{~cm}$ (10th percentile), respectively. The physical examination of the patient with respiratory distress showed that he had underdeveloped midface, a flattened forehead, epicanthal fold, anteverted nares, small nose, depressed nasal bridge, small mouth, narrow palate, pectus excavatum, hydrocele, and bulbous fingertips (Fig. 1). He was born by normal spontaneous vaginal delivery at the 40th gestational week as the first living child (first pregnancy) of a healthy 17-yearold mother who was married to a healthy 23 -year-old father. The mother and father of the patient were first-degree cousins. Family history was unremarkable. Maxillofacial computerized tomography showed bilateral severe choanal stenosis and calcification in the nasal septum. Stents were placed by an otorhinolaryngologist in both nostrils of the baby who had severe respiratory distress and therefore could not be fed orally. Respiratory distress improved and oral feeding was possible. Complete blood count and biochemical examina- tion results were normal. There was no significant pathological finding on echocardiographic examination. The abdominopelvic ultrasound result was normal, whereas transfontanellar ultrasound revealed calcification, compatible with hyperechogenic lesions around periventricular basal ganglia. The noncontrast brain CT showed that there were numerous microcalcifications in both hemispheres (Fig. 2A). The toxoplasmosis, rubella, cytomegalovirus, and herpes simplex virus serology result was negative. In the bone survey, an increase was observed in diffuse sclerosis in the vertebral bodies and long bones (Fig. 2B). Auditory brainstem response test was performed, and the infant failed to pass the test. The patient, who presented with the characteristic features of RS and a novel homozygous c.1255T >C (p.W419R) variant in the FAM20C gene, was diagnosed with nonlethal RS. The patient was not given any treatment other than 400 IU supplementary vitamin D per day. The last control of the patient was made when he was 12 months old. He had no complaints. The patient's height was $75 \mathrm{~cm}$ (25th-50th percentile), weight was $8.3 \mathrm{~kg}$ (3rd-10th percentile), and head circumference was $45.5 \mathrm{~cm}$ (10th-25th percentile). The patient was breathing comfortably, and his physical examination findings were normal. The patient's complete blood count and biochemical examinations such as calcium, phosphorus, and alkaline phosphatase were within normal limits. There was no fracture in the bones of the patient and there was no finding other than osteosclerosis in the bone radiographs of the patient (Fig. 3).

\section{Materials and Methods}

\section{Cytogenetic Analysis}

Chromosomal analyses were performed on peripheral blood samples using conventional G-banding techniques (550-band resolution). Results were reported according to the International System for Human Cytogenomic Nomenclature [ISCN, 2016]. Standard karyotyping showed no visible numerical or structural alter- 
Fig. 3. Lateral (A) and anteroposterior (B) direct radiographs at the age of 12 months. Osteosclerosis was observed only in the laterals of the bilateral ribs and at the costovertebral joints.
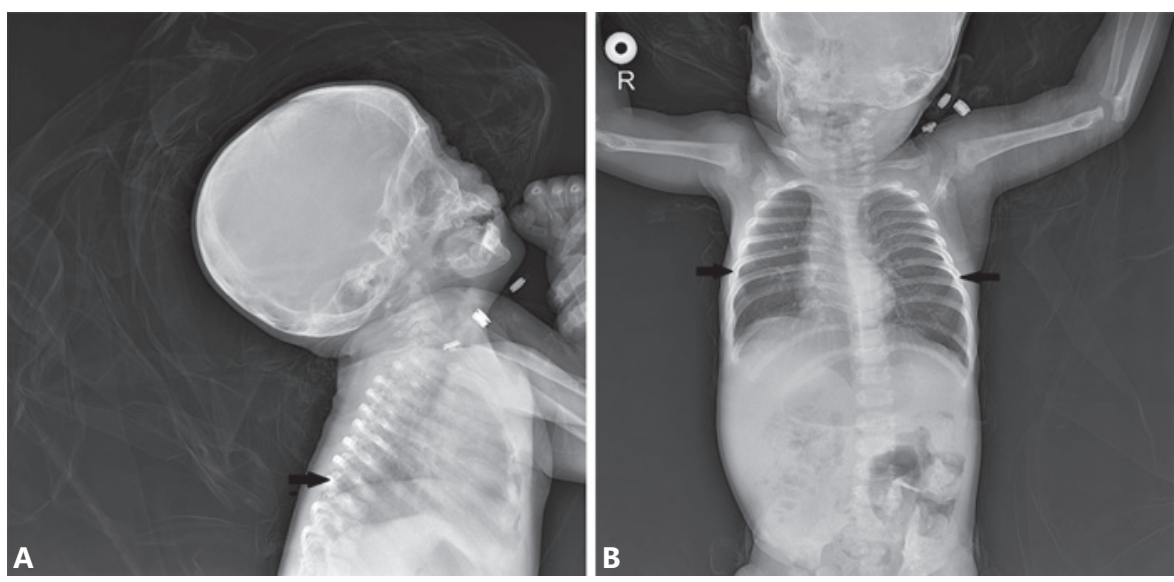

ations in chromosomes. The patient showed male karyotype $(46, \mathrm{XY})$.

\section{Molecular Genetic Analysis}

Genomic DNA was extracted from peripheral blood leukocytes using the QIAamp DNA Blood Midi Kit (Qiagen, Hilden, Germany). In the present study, the MiSeq next-generation sequencing platform (Illumina Inc., San Diego, CA, USA) was used to perform the FAM20C gene sequence analysis. Sequencing data analysis was performed using MiSeq Reporter software (Illumina Inc.). Human genome assembly 19 (hg19) was used as the reference. Alignment to the hg19 genome and variant calling was performed using MiSeq Reporter Software (Illumina Inc.). Aligned sequence reads were visualized with IGV 2.3 (Broad Institute, Cambridge, MA) software.

\section{Results}

Anovelhomozygousvariantc.1255T $>C$ (p.Trp419Arg) in exon 7 of the FAM20C gene was identified in the proband. The healthy parents were heterozygous carriers for the same variant. No records regarding the new variant were found in the HGMD, DbSNP, or ClinVar databases, or in OMIM, Google, or PubMed. The novel unpublished variant was evaluated using in silico tools (MutationTaster, PolyPhen-2, Provean, SIFT, Revel, and PrimateAI). The variant was classified as likely pathogenic based on the recommendations of the American College of Medical Genetics [Richards et al., 2015].

\section{Discussion}

The majority of mutations identified in patients with both lethal and nonlethal RS are missense mutations, whereas nonsense, complex, splicing mutations, deletion, and frameshift insertion have been reported rarely [Mamedova et al., 2019]. The FAM20C mutations were suggested to affect protein secretion by impairing protein stability and folding kinetics or to cause loss of function in kinase activity by affecting catalytic or nucleotide binding sites [Faundes et al., 2014; Mamedova et al., 2019]. These mutations have been observed to cause growth retardation as well as defects in the growth plate and osteoblast differentiation. In the present study, we reported a male case with nonlethal RS who had a novel missense c.1255T $>C$ (p.W419R) homozygous variant, choanal stenosis, osteosclerosis, intracerebral calcification, and dysmorphic facial features. To the best of our knowledge, the variant detected in this case with a milder phenotype has not been previously reported in the literature. We believe that this variant may explain the milder phenotype due to the nature or localization of the substitution within the protein. As with all rare syndromes, it is difficult to determine a direct genotype-phenotype correlation due to the small number of diagnosed patients and the different mutations encountered in these patients. A summary of the laboratory parameters and the clinical findings in 5 patients reported in the literature and present case is shown in Table 1 .

FAM20C, which is thought to have a very important role in normal bone development, is highly expressed in mineralized tissues [Ababneh et al., 2013]. FAM20C encodes a Golgi casein kinase that phosphorylates secretory calcium-binding phosphoproteins [Acevedo et al., 2015]. Mineralized tissue diseases and ectopic mineralization develop when the phosphorylation status of these proteins is impaired [Giachelli, 2005]. Inactivation of the FAM20C gene in patients with RS is thought to impair phosphorylation, causing an increase in bone density and an increase in mineralization [Takeyari et al., 2014]. Similar to the cases in the literature [Raine et al., 1989; Simpson et al., 2009; 


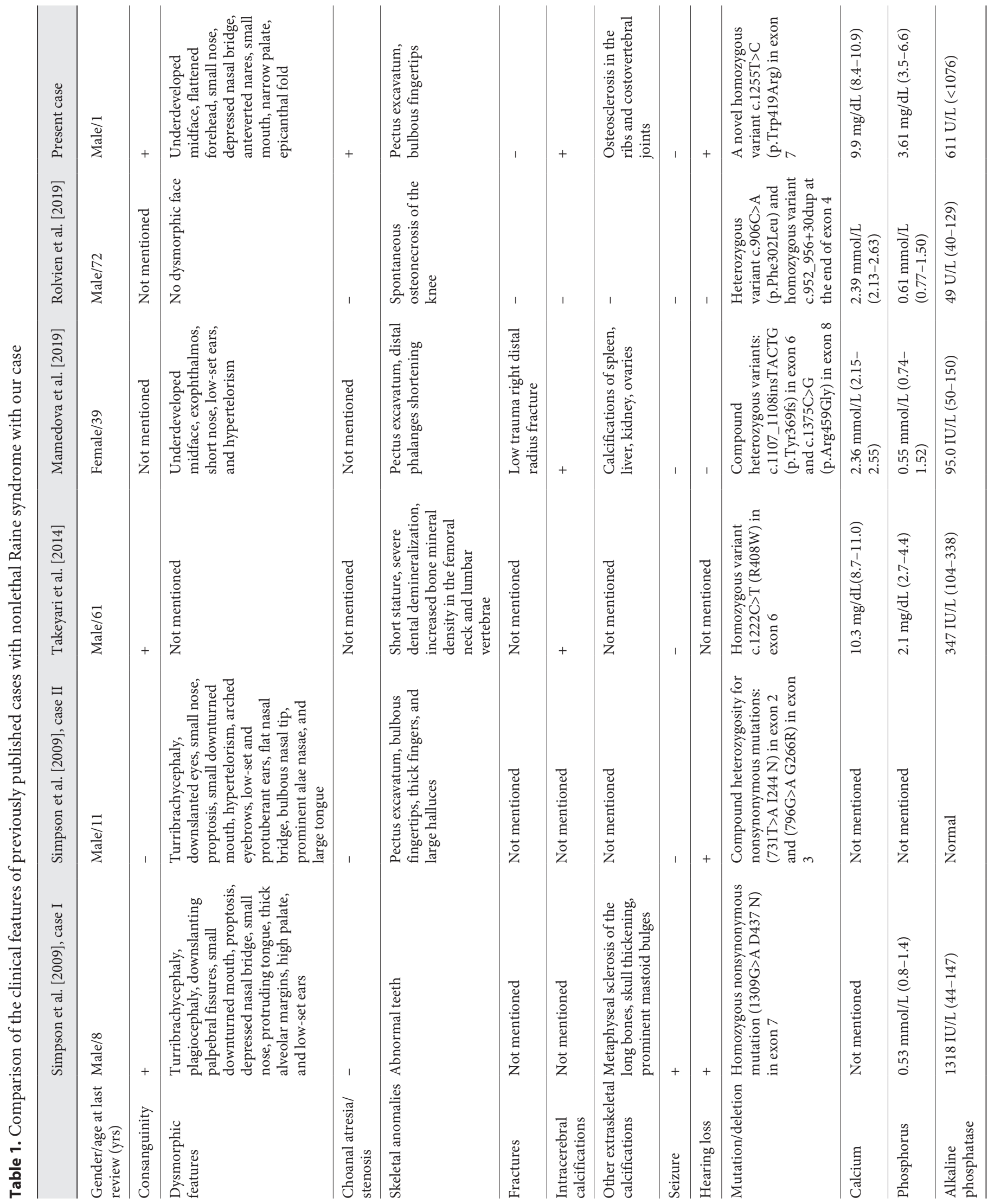


Ababneh et al., 2013; Mamedova et al., 2019; Eltan et al., 2020], intracerebral calcification and osteosclerosis in the vertebral corpus and long bones were observed due to increased bone density and mineralization in the present case. Characteristic facial features occurring as a result of increased ossification in the facial and skull bones, such as proptosis of the eyes, narrow prominent forehead, underdeveloped midface, and depressed nasal bridge, have been reported in this syndrome [Simpson et al., 2009]. The present case also had dysmorphic features such as midface hypoplasia, small nose, depressed nasal bridge, small mouth, and narrow palate; however, the features were milder than previously published cases with nonlethal RS [Simpson et al., 2009; Mamedova et al., 2019]. Other clinical findings seen in patients with nonlethal RS include hydrocephalus, microcephaly/brachycephaly, pectus excavatum, choanal atresia/stenosis, and hearing loss [Simpson et al., 2009; Acevedo et al., 2015; Mamedova et al., 2019]. Choanal stenosis, pectus excavatum, and hearing loss were also detected in the present case. On the other hand, a 72-year-old man who was diagnosed with mild RS only had spontaneous knee osteonecrosis, phosphate loss, and a significant trabecular high bone mass, but no dysmorphic findings [Rolvien et al., 2019].

There is a need for further studies involving a larger number of patients for a better understanding of molecular pathogenesis and better genotype-phenotype correlation. Furthermore, we believe that identifying the clinical characteristics of individuals with RS may facilitate the diagnosis and follow-up of these patients in the community. The increase in the number of case reports focusing on these syndromes will contribute to the understanding of how these disorders develop and progress and increase the awareness of rare diseases.

\section{Acknowledgement}

The authors wish to thank the patient and his family for their participation. We would like to also thank Intergen Laboratory Group for molecular genetic analysis and Professor Nurcan Aras for cytogenetic analysis.

\section{Statement of Ethics}

Informed consent for genetic analysis and publication of clinical reports and photographs were obtained from the patient's parents in compliance with the national ethics regulation.

\section{Conflict of Interest Statement}

The authors have no conflicts of interest to disclose.

\section{Funding Sources}

No specific funding was obtained from any agency or organization for the current study.

\section{Author Contributions}

N.E. performed the research including data collection. N.E and Y.C. produced the first draft of the manuscript. Both authors reviewed, edited, and approved the final version of the submitted manuscript.

\section{References}

Ababneh FK, AlSwaid A, YoussefT, Al Azzawi M, Crosby A, AlBalwi MA. Hereditary deletion of the entire FAM20C gene in a patient with Raine syndrome. Am J Med Genet A. 2013; 161A(12):3155-60.

Acevedo AC, Poulter JA, Alves PG, de Lima CL, Castro LC, Yamaguti PM, et al. Variability of systemic and oro-dental phenotype in two families with non-lethal Raine syndrome with FAM20C mutations. BMC Med Genet. 2015; 16:8.

Eltan M, Alavanda C, Yavas Abali Z, Ergenekon P, Yalındag Ozturk N, Sakar M, et al. A Rare Cause of Hypophosphatemia: Raine Syndrome Changing Clinical Features with Age. Calcif Tissue Int. 2020;107(1):96-103.

Faundes V, Castillo-Taucher S, Gonzalez-Hormazabal P, Chandler K, Crosby A. Chioza B Raine syndrome: an overview. Eur J Med Genet. 2014;57(9):536-42.
Giachelli CM. Inducers and inhibitors of biomineralization: lessons from pathological calcification. Orthod Craniofac Res. 2005;8(4):229-31.

ISCN 2016. An International System for Human Cytogenomic Nomenclature. Cytogenet Genome Res. 2016;149(1-2):1-140.

Mamedova E, Dimitrova D, Przhiyalkovskaya E, Buryakina S, Vasilyev E, Tiulpakov A, et al. Non-lethal Raine Syndrome in a Middle-Aged Woman Caused by a Novel FAM20C Mutation. Calcif Tissue Int. 2019;105(5):567-72.

Raine J, Winter RM, Davey A, Tucker SM. Unknown syndrome: microcephaly, hypoplastic nose, exophthalmos, gum hyperplasia, cleft palate, low set ears, and osteosclerosis. J Med Genet. 1989;26(12):786-8.

Richards S, Aziz N, Bale S, Bick D, Das S, GastierFoster J, et al. Standards and guidelines for the interpretation of sequence variants: a joint consensus recommendation of the American
College of Medical Genetics and Genomics and the Association for Molecular Pathology. ACMG Laboratory Quality Assurance Committee. Genet Med. 2015;17:405-24.

Rolvien T, Kornak U, Schinke T, Amling M, Oheim R. A novel FAM20C mutation causing hypophosphatemic osteomalacia with osteosclerosis (mild Raine syndrome) in an elderly man with spontaneous osteonecrosis of the knee. Osteoporos Int. 2019;30(3):685-9.

Simpson MA, Scheuerle A, Hurst J, Patton MA, Stewart H, Crosby AH. Mutations in FAM20C also identified in non-lethal osteosclerotic bone dysplasia. Clin Genet. 2009;75(3):271-6.

Takeyari S, Yamamoto T, Kinoshita Y, Fukumoto S, Glorieux FH, Michigami T, et al. Hypophosphatemic osteomalacia and bone sclerosis caused by a novel homozygous mutation of the FAM20C gene in an elderly man with a mild variant of Raine syndrome. Bone. 2014;67:56-62. 